\title{
Affekt, Genuss und das Problem der Mentalisierung. Elemente einer Sozialpsychologie des rechten Populismus
}

\author{
Reinhold Görling
}

\section{Political Correctness}

In einem der Videos aus dem Jahre 2016, das die New York Times noch vor der Wahl des gegenwärtigen US-amerikanischen Präsidenten Trump publiziert hat, ist ein vielleicht vierzigjähriger Mann am Rande einer Wahlveranstaltung zu sehen. Mit heftigen Stoßbewegungen seiner Hüfte skandiert er mehrfach „Fuck political correctness! Fuck political correctness!..." Dabei wendet er sich im Halbkreis, so als suche er überhaupt erst einen Adressaten für seine verbalen und körperlichen Äußerungen. Im Video folgen dann mehrere Beispiele misogyner Beschimpfungen der demokratischen Präsidentschaftskandidatin Hillary Clinton, dem voran gingen Szenen, die zeigen, wie Zuhörer*innen frenetisch dem Versprechen Trumps applaudieren, eine Mauer zwischen Mexiko und den USA zu errichten, wenn er gewählt werde (Parker/Corasaniti/Berenstein 2016).

Diese Aufnahmen sind Beispiele für eine intensive Sexualisierung des politischen Raumes und für eine dabei stattfindende enge Verquickung von sexuellen Fantasien mit solchen der Macht und Bemächtigung. Diese Fantasien der Bemächtigung sind deutlich durch Geschlechterbilder geprägt, die Bemächtigung ist zugleich eine Erniedrigung und symbolische Feminisierung des Anderen. Die eine Penetration simulierenden Hüftbewegungen des Mannes gelten einem politischen Begriff, einer sprachlichen, eher abstrakten diskursiven Wendung. Sie wird durch diese sexuellen Handlungen allerdings verkörpert. Wie ist eine solche Verknüpfung von sexueller Erregung und Sprache zu verstehen?

Der Begriff Political Correctness ist seit etwa 1990 in den USA zu einem Kampfbegriff der Konservativen und der politischen Rechten geworden. Sein

R. Görling $(\bowtie)$

Berlin, Deutschland

E-Mail: reinhold.goerling@hhu.de 
Ursprung dürfte in den 1970er Jahren zu suchen sein. Er kam damals eher ironisch in linken Diskussionen über die richtige politische Linie in Umlauf, wurde dann aber im darauf folgenden Jahrzehnt zunehmend $\mathrm{zu}$ einem identitätspolitischen Begriff, der von Gruppen benutzt wurde, die sich einer sozialen Benachteiligung ausgesetzt sahen, historisch und gegenwärtig (Weigel 2016). Im Zentrum steht dabei die Forderung nach Anerkennung und Gleichbehandlung von Menschen, die aufgrund einer kulturellen, ethnischen oder sexuellen Differenz sozialer Diskriminierung ausgesetzt sind. Da Namen und sprachliche Wendungen mit solchen Diskriminierungen historisch verbunden waren und gegenwärtig verbunden sind, konzentrierte sich die Diskussion stark auf die Ebene der Bezeichnungen und der diskursiven Praktiken. Damit ist allerdings nicht garantiert, dass die spezifischen Verknüpfungen von Sprache und Körper und ihre Bewertung, die sich historisch ergeben hat, schon aufgelöst wurde. Habitus, Geschlecht und Hautfarbe bleiben in der Regel markiert, nur dass dies in neuen sprachlichen Wendungen nun oft verschoben und damit schwerer adressierbar wird. Auch können diese Wendungen die Verfangenheit in den Strukturen der Machtkämpfe seitens der Gruppen, die um Anerkennung kämpfen, zu verdecken helfen.

Um die sprachliche Problematik, die mit der Forderung nach ,politisch korrekter' Adressierung verbunden ist, besser zu verstehen, macht es Sinn, zwei sich überlagernde Ebenen zu unterscheiden. Die Dimensionen der sozialen Praktiken, die mit der Ausübung von Macht über andere verbunden sind, können durchkreuzt werden von Praktiken, die das Verhältnis von Nähe und Distanz verhandeln. Und dabei können durchaus Reibungen und Widersprüche entstehen. Für Menschen, die sich aufgrund bestimmter Merkmale Diskriminierungen ausgesetzt sehen, versprechen wertneutrale Formulierungen einen gewissen Schutzraum zu bieten. So stellte etwa der US-amerikanische Filmtheoretiker Robert Stam in einem längeren Symposiumsbeitrag, der 1992 erschien, fest, dass die geforderte Standardisierung der Etikette auch zu einer Distanzierung zwischen den Gruppen führe: „,Thus ,political correctness “ is premised on distance and a lack of intimacy“ (Ross 1993: 35). Die soziale und politische Folge ist aber selbst wiederum ambivalent, denn das, was aus der Perspektive derer, die von sprachlichen Praktiken diskriminiert wurden, als ein Minimum von Anerkennung erfahren werden kann, das überhaupt erst eine Begegnung ermöglicht, kann von denen, die aus der Diskriminierung und der in ihr impliziten malignen Intimität bisher ein unproblematisiertes Selbstbewusstsein bezogen haben, als ein Entzug erfahren werden. Jedenfalls löst die Forderung nach Political Correctness die phantasmatische Intimität zwischen den gesellschaftlichen Gruppen nicht schon auf, oft verschiebt sie sie nur. Sie wird dann nicht mehr in den Ansprachen und Praktiken direkt verhandelt, sondern entstellt, gewissermaßen an einem Deckphänomen. Das macht ihre Adressierung und Symbolisierung keineswegs einfacher. Und es schafft ein neues Unausgesprochenes, das wiederum vonseiten derer, die bislang am Genuss der Diskriminierung partizipierten, aufgegriffen und projektiv als Vorwurf der Zensur zurückgewendet werden kann.

Eines der ersten Dokumente, in denen diese typischen Verdrehungen des Arguments beobachtet werden können, ist ein Artikel von Richard Bernstein, der am 
28. Oktober 1990 in der New York Times erschien. Tatsächlich bezieht sich der Autor, der damals der Redaktion der Tageszeitung angehörte, anfangs auch auf den ursprünglich ironischen Sinn des Begriffs, verweist dann aber darauf, dass er mehr und mehr in Debatten darüber in Gebrauch käme, was an den Universitäten gelehrt werden sollte:

„There are even initials - p.c.p. - to designate a politically correct person. And though the terms are not used in utter seriousness, even by the p.c.p.'s themselves, there is a large body of belief in academia and elsewhere that a cluster of opinions about race, ecology, feminism, culture and foreign policy defines a kind of ,correct" attitude toward the problems of the world, a sort of unofficial ideology of the university“ (Bernstein 1990). ${ }^{1}$

Er werde, so Bernstein weiter, inzwischen aber auch spöttisch als „sarcastic jibe“ von Konservativen und traditionellen Liberalen aufgegriffen, mit dem Ziel ,to describe what they see as a growing intolerance, a closing of debate, a pressure to conform to a radical program or risk being accused of a commonly reiterated trio of thought crimes: sexism, racism and homophobia“ (ebd.). Am 4. Mai 1991, also etwa ein halbes Jahr später, hielt der damalige US-amerikanische Präsident George H.W. Bush an der University of Michigan eine Rede, die dieses Argument aufgriff und bis hin zum Vorwurf der Zensur erweiterte:

„The notion of political correctness has ignited controversy across the land. And although the movement arises from the laudable desire to sweep away the debris of racism and sexism and hatred, it replaces old prejudice with new ones. It declares certain topics offlimits, certain expression off-limits, even certain gestures off-limits“ (Bush 1991).

Es ist nicht ohne weiteres diskursiv nachvollziehbar, wieso eine Tabuisierung bestimmter Wendungen, Ausdrücke und Gesten zu einer Ersetzung von alten durch neue Vorurteile führen kann, wenn eben diese zuvor Ausdruck von Vorurteilen waren. Es lässt sich wohl nur so verstehen, dass die Rede über ein off-limit, über etwas, das tabuisiert ist, eben das Nichtbezeichnete aktualisiert, phantasmatisch hervorruft: racism, sexism, hatred. Trumps Kampagne von 2016 geht nun aber einen Schritt weiter. Unter Berufung auf das Recht zur freien Meinungsäußerung wird dieses Tabu gezielt gebrochen. Das ist nun nicht einfach eine Rückkehr zu alten Sprechweisen. Was Trump und seine Anhänger*innen performativ vorführen, ist ein Genuss an der Diskriminierung anderer, der sich ohne Angst vor Strafe realisieren lässt, weil er nun im Namen des Rechts zu geschehen scheint. Die Sexualisierung und die Realisierung von Gewalt im Eindringen in den Schutz- oder Intimraum des Anderen geschehen im Licht der Scheinwerfer und vor den Objektiven der Kameras. Sexismus, Rassismus und Homophobie werden nicht mehr nur benannt, sondern offensiv verbal ausagiert. Der politische Raum wird zu einem offenen Schauplatz sexueller und aggressiver Gefühle.

\footnotetext{
${ }^{1}$ Es liegt nahe, dass Bernstein mit dieser Abkürzung auch auf das als Droge bekannte Dissoziativum Phenylcyclohexylpiperidin (PCP) anspielt. (Ich danke Christina Rogers für diesen Hinweis.).
} 
Es sind Affekte ohne Objekt, Affekte der Wut, der unbestimmten Angst, denen nun politisch vorgeformte Genres oder Szenen angeboten werden. Diese Genres repräsentieren nicht, sie können kaum in diskursive Praktiken über die Erfahrung der Welt und ihrer Dinge und Objekte überführt werden. Sie dienen als Szenen der Beschimpfung oder, wie im Beispiel von Trumps wall, des Jubels. Ob politische Gegner als Krüppel, Frauen als willfähriges Sexualobjekt oder ethnisch differente Gruppen mit Analmetaphern adressiert werden: Diese Kampagne der sarkastischen Verhöhnung der Idee der Political Correctness ist vor allem als Umkehrung der in ihr einmal geforderten Distanzierung zu verstehen. Es geht weniger um die Übertretung politischer Grenzen als um die Transgression sozialer und psychischer Schutzräume, um das Eindringen in die Intimsphäre des Anderen. Das produziert ein Gefühl männlicher Bemächtigung. Die Sexualisierung der Politik ist genau genommen ein Angriff auf den Schutzraum der Intimität des oder der Anderen. Das kann aufseiten der Opfer zu einer Art Lähmung führen, da eben dieses Eindringen in den Schutzraum in der Regel Scham bei denjenigen hervorruft, die so verletzt werden. Scham ist ein Versuch, eine bedrohte Integrität vor einer Auflösung zu retten.

Diese Sexualisierung des Politischen und die Verkörperung des Anderen mittels der eigenen verbal und körperlich ausgespielten Gewalt muss man als einen obszönen Triebgenuss verstehen. Obszön ist er auch deshalb, weil er das eigene Begehren in dem Maße verleugnet, wie er es artikuliert. Schon Max Horkheimer und Theodor W. Adorno hatten in den „Fragmenten zum Antisemitismus“, die sie in der Dialektik der Aufklärung publizierten, darauf verwiesen, dass der Rassenhass in aller Regel mit einem mimetischen Begehren des Anderen verbunden ist: kein Antisemit, „dem nicht im Blute läge, nachzuahmen, was ihm Jude heißt“ (Horkheimer/Adorno 1983: 193). Während diese Beobachtung noch im Vorfeld der manifesten Verfolgung und Ermordung der Jüd*innen in Europa durch die Nationalsozialist*innen und ihre Verbündeten in den besetzten Ländern zu situieren ist, muss die eigentliche Verfolgung dann auch als sexualisierte Gewalt begriffen werden. Dem widerspricht gerade nicht, dass die gesuchte Nähe mit einer absoluten Ausgrenzung gekoppelt wird: ,That wall! Fuck those bloody beaners!“ (Parker/Corasaniti/Berenstein 2016).

\section{"Ins Gesicht gefilmt" - Öffentliche Intimität}

Dem erwähnten Videodokument soll noch ein zweites zur Seite gestellt werden, das eine Handlung zeigt, die im Spätsommer 2018 in Deutschland Gegenstand politischer Debatten wurde. Ein Mann mit einem Stoffhut in den Farben der deutschen Nationalfahne nimmt an einer Demonstration von Pegida in Dresden teil. Als er ein für das ZDF arbeitendes Reporterteam bemerkt, beschimpft er den Kameramann und behauptet, dieser beginge eine Straftat, weil er Aufnahmen von ihm aus der Nähe mache. ,Sie haben mich ins Gesicht gefilmt, das dürfen Sie nicht. Frontalaufnahme. Sie haben eine Straftat begangen!“ Ein zweiter Mann in einem grünen T-Shirt tritt hinzu und macht Kreisbewegungen mit ausgestrecktem 
Arm auf die Kamera zu, die zumindest symbolisch seine Forderung unterstreichen und den Eindruck erwecken, als wolle er den Kameramann ohrfeigen: „Mach die Kamera weg! Mach die Kamera weg!“ Es ist so, als sei die Kamera ein Schmutz, den ein Vater auf seiner Jacke trägt, oder eine fremde Maske, die ein Kind bedroht. Schließlich mischt sich eine etwa fünfzig Meter entfernt stehenden Gruppe von Polizisten ein und hält die Reporter*innen, nicht aber die Aggressor*innen, für einige Minuten fest und überprüft ihre Personalien, sogar mehrfach (Schmidt/ Schiele 2018).

Es geht auch hier vor allem um die starken Emotionen, mit denen der Mann mit der Mütze in den Farben der Nationalflagge agiert, weniger darum, dass die Polizisten eine erschreckende Unkenntnis der Rechte der Presseorgane zeigten oder dass der pöbelnde Mann Mitarbeiter des sächsischen Landeskriminalamtes war, wie sich bald herausstellen sollte. „Sie haben eine Straftat begangen!“ Der Mann reklamierte nicht das Recht auf die Selbstbestimmung über sein Bild, das ja bekanntlich begrenzt ist in dem Augenblick, an dem man an einer öffentlichen politischen Aktion teilnimmt. Er empörte sich, in seiner Erregung zunehmend errötend, über ein angebliches Vergehen, das in nichts anderem als in der Herstellung von Öffentlichkeit bestand, obwohl es sich ja um eine öffentliche und sich an die Öffentlichkeit richtende Veranstaltung handelte. Das deutet zunächst auf eine merkwürdige Einengung der Vorstellung des Politischen und der Öffentlichkeit hin. Es wird nicht als Raum der diskursiven Auseinandersetzung verstanden, sondern als Möglichkeit einer Inszenierung eines Wir, das sich selbst in dem Maße schafft, in dem es ein Außen adressiert. Wenn Beistehende in dieser Situation die als Schimpfwort verstandene Wendung „Lügenpresse“ skandieren, scheinen sie die Rechtfertigung für ihr in der Demonstration inszeniertes Wir gefunden zu haben.

Es gibt jedoch auch eine merkwürdige Überlappung zwischen der Anrufung eines sexuellen Aktes der Penetration des Teilnehmers während der Wahlkampfveranstaltung Trumps und der Imagination einer vollzogenen Penetration, ,ins Gesicht" gefilmt worden zu sein, seitens des Teilnehmers der Pegida-Demonstration. Die Fantasie der Übertretung äußert sich im zweiten Falle nur in einer passiven Wendung. Man könnte vermuten, dass die Demonstration selbst zunächst als eine Inszenierung der eigenen Macht erfahren, dass dies aber durch die Anwesenheit der Kamera dann gestört wird. Es ließe sich also etwas über die Intensität der Gefühle in Erfahrung bringen, welche die Demonstration bei diesem Teilnehmer hervorruft: Gefühle einer souveränen Übertretung, eines Raums der Allmacht, in den nun tatsächlich, wie in einen Intimbereich, die Kamera tritt.

Doch erklärt das nicht, warum das Reporterteam überhaupt angesprochen wird. Tatsächlich suchen beide Personen regelrecht den Adressaten ihrer Performance. Im Fall des Mannes in Dresden verhält es sich so, dass er aus einer Distanz von vielleicht zwanzig Metern auf den Kameramann zugeht und ihn beschimpft, obwohl ja erst durch diese Handlung eine körperliche Nähe zur Kamera hergestellt wird. Unter Beachtung der kurzen Brennweite, mit der die Aufnahmen gemacht sind, erscheint die massiv reduzierte Entfernung zwischen dem Mann und seinem affektiv erregt adressierten Gegenüber jetzt tatsächlich so etwas wie 
ein körperliches Eindringen in einen privaten oder gar schon intimen Bereich zur Folge zu haben. Es geht hier wie dort um eine intensive Aufladung und Übertretung der Differenz oder Grenze zwischen einem Innen und einem Außen, einem Intimen und einem Öffentlichen, einem Eigenen und einem Fremden.

\section{Infrastruktur der Affekte}

In seinem Dokumentarfilm Fahrenheit 11/9 (2018) hat Michael Moore eine Reihe von Filmaufnahmen aus Wahlkampfveranstaltungen Trumps aufgenommen, die Szenen zeigen, in denen Menschen, die wegen ihrer nicht-, weißen' Hautfarbe auffallen, verhöhnt, bespuckt, geschlagen werden. Dabei fordert Trump seine Zuhörer*innen zur Gewalt auf: „Look at my Afro-American over there! Look at him!“ (Moore 2018: 0.34:25) Und dann wechselt er mit ausgestrecktem Arm und Zeigefinger in die direkte Ansprache dieser einen Person: „Go home and get a job, go home, get a job!“ Woraufhin zu sehen ist, wie ein Mann mit einem kleinen Plakat bespuckt und aus dem Saal hinausgestoßen wird. Bei einer anderen Gelegenheit sagt Trump, Sicherheitspersonal hätte ihm mitgeteilt, dass Anwesende möglicherweise Tomaten mitgebracht hätten. Falls die Zuhörer*innen jemanden mit Tomaten sehen sollten, so Trump weiter: „Knock the crap out of him. I go and pay for the legal fees, I promise." Aber diese Gebühren wären sowieso nicht hoch, weil die Gerichte eigentlich dafür da seien, dass so etwas geschieht (ebd.: 0.34:40). Bekannt geworden ist auch die Rede im Januar 2016 im Sioux Center in Iowa, in der Trump sich brüstet, so kluge Anhänger*innen zu haben, dass sie ihm vollständig vertrauten: „I could stand in the middle of Fifth Avenue and shoot somebody and I wouldn't loose any voters, O.K.? That's like incredible" (Diamond 2016). Trump beschreibt damit eine Szene, in der er selbst als Souverän auftritt, der in aller Willkür über Leben und Tod anderer Menschen entscheidet. Und er scheint zu wissen, dass gerade das etwas ist, das seine Anhänger*innen an ihm bewundern. An der Ermächtigung, die Trump inszeniert, können seine Anhänger*innen imaginär teilhaben - und das selbst dann, wenn sie eigentlich Opfer sind. Letzteres gilt etwa für die vielen Frauen in seiner Wählerschaft, die sich von seinen sexistischen und misogynen Äußerungen in ihrer Bewunderung nicht irritieren lassen. Michael Moore nimmt hier noch eine weitere Dimension von Trumps öffentlichen Auftritten auf: sein Verhältnis zu seiner Tochter Ivanka. Er gibt mehrere Fotografien wieder, auf denen Tochter und Vater in Umarmungen vor der Kamera posieren, die einem Ehe- oder Liebespaar entsprechen. Und auch mehrere Talk-Show-Auftritte, in denen Trump ein auf seine Tochter gerichtetes sexuelles Begehren offen artikuliert: Auf die Frage einer Moderatorin an Ivanka Trump „What is the favorite thing you have in common with your father?" antwortet diese: „Either real estate or golf.“ - „Donald, with your daughter?“ - „Well, I was going to say sex but I cannot relate that." Der Satz wird mit einer körperlichen Geste der Zuwendung zu seiner Tochter beendet (ebd.: 0.21:05-0.23:05). 
In einem kurz nach der Wahl Trumps in Social Text erschienenen Essay stellt Lauren Berlant einen Zusammenhang zwischen der Geste der ungebrochenen Souveränität und dem Bezug zur Welt und ihren Objekten her (Berlant 2017). Das Phantasma der Souveränität kennt keine Vermittlung, das lässt sich den Theorien der kolonialen Gewalt, wie sie Frantz Fanon und mit Bezug auf ihn Achille Mbembe entwickelt haben, entnehmen. ${ }^{2}$ Für den Souverän gibt es keine Anerkennung des anderen Subjekts, es wird für ihn zu einem Objekt, das wie jedes Ding seiner Autonomie beraubt ist. Es gibt für den Souverän keine Arbeit am Anderen oder Fremden. Sprache ist für das Phantasma der Souveränität damit in einem wesentlichen Sinne keine Repräsentation einer unabhängigen Welt, über deren Bedeutung zu diskutieren wäre, sondern stellt so etwas wie einen ausgestellten Bezug der Bemächtigung von Welt her. Die Worte ersetzen die Welt der Objekte, was sie aber auch dazu gebrauchen lässt, in ihnen sehr direkt Affekte auszuagieren, zu genießen.

Selbstverständlich können Worte immer eine affektive Besetzung erlauben. Vor allem die poetische Sprache zeichnet so etwas aus, doch hier wissen wir um die Differenz zwischen Sprache und Welt, ja, die poetische Sprache öffnet gerade diese Differenz und löst dadurch die alltäglichen Verknüpfungen. Die alltäglichen Verknüpfungen von Worten und Dingen mit Affekten vollzieht sich durch ihre Integration in Szenen und Narrative. Damit binden wir uns an die Welt oder lassen uns von ihr halten. Diese Szenen und ihre Genres nennt Berlant die Infrastruktur, in der wir leben (Berlant 2016: 394). Sie ist grundsätzlich prekär und veränderlich, aber wir klammern uns auch an sie, wie Berlant in ihrem Buch Cruel Optimism analysiert (Berlant 2011). In ihrem Essay zu Trump bezieht sie diese Infrastruktur der affektiv besetzten Szenen auch auf den Begriff der commons, der Gemeinschaft. Während es ein philosophisches Konzept der Gemeinschaft gebe, nach dem diese Infrastruktur als Öffnung für ein Kommendes verstanden wird, wie sie in offensichtlichem Bezug zur Theorie der Gemeinschaft von JeanLuc Nancy schreibt, ohne dessen Namen allerdings zu erwähnen, gebrauche die Rechte das Konzept der Gemeinschaft gewissermaßen als Schließung einer Bewegung, in der die Bindung zur Welt zunächst gebrochen wird. Im Zuge dieser Bewegung werde zudem offensichtlich, wie sehr wir uns an Repräsentationen klammern, die uns die Solidität der Welt zeigen sollen. Trumps Geste des Souveräns und seine willkürlichen Äußerungen und Entscheidungen offerieren die Haltlosigkeit aller Vorstellungen der Verlässlichkeit unserer Beziehungen zur Welt. „The world is a mess. The world is as angry as it gets, “ wird Trump dann

\footnotetext{
${ }^{2}$ Fanons Kritik am Phantasma der Souveränität ergibt sich aus seiner Analyse der kolonialen Herrschaft als einer Gewalt ohne Vermittlung, was ihn in kritischem Bezug auf die an Hegels Dialektik orientierten Theorien der Emanzipation zur Formulierung führt, dass im antikolonialen Kampf Gewalt als „die ideale Vermittlung“ angesehen werden muss. (Fanon 1981: 72). Zum Verständnis dieser Denkbewegung vgl. das Kapitel „La pharmacie de Fanon“ aus Mbembes Politiques de l'inimitié (Mbembe 2016: 91-140); zu Mbembes Kritik der Souveränität vgl. seinen Aufsatz „Necropolitics“ (Mbembe 2003).
} 
auch kurz nach Übernahme des Amtes in mehreren Interviews verkünden (Trump 2017). Die sozialpsychologische Logik, auf die Berlant verweist, besteht nun darin, dass, haben wir erst einmal diese Repräsentation der Welt aufgegeben, eine Empfänglichkeit für neue Strukturen wächst: „,we can make better infrastructures for coordinating our proximities and reproducing life, by which I mean lives but also life, the rhythms of ongoingness for which we show up because we wake up" (Berlant 2017). Je schwächer die Verlässlichkeit der Vorstellung von der Welt , da draußen' ist, um so leichter lässt sich ein affektiver und emotionaler Innenraum herstellen, je schwächer die Verlässlichkeit der Welt erscheint, um so größer wird die Figur eines Mannes, der in einer Geste der Willkür seinen eigenen élan vital, wie Berlant mit Bezug auf Henri Bergson sagt, ausstellt (ebd.).

\section{Genuss und Verleugnung}

Im Folgenden soll es vor allem darum gehen, die Dynamik genauer zu beschreiben, die zwischen dem erfahrenen Mangel gegenüber einer Welt, deren Objekte und Bilder keine Verlässlichkeit mehr bieten oder nie geboten haben, und der Sexualisierung des öffentlichen Raumes besteht, oder anders gesagt, die zwischen dem Zusammenbruch einer Infrastruktur und dem Genuss der Willkür besteht. Schauen wir noch einmal auf das gängige psychoanalytische Erklärungsmuster. In der älteren psychoanalytischen Tradition werden Rassismus, Sexismus, Homophobie und andere Formen der Missachtung und Gewalt gegenüber dem Anderen meist als ein Gefüge von Faszination und Abwehr begriffen. Nehmen wir als Beispiel die schon erwähnte, klassisch zu nennende Theorie über „Elemente des Antisemitismus“, wie sie Max Horkheimer und Theodor W. Adorno noch vor Ende des Zweiten Weltkrieges in ihrer Dialektik der Aufklärung formuliert haben. Die andere Person ruft ein sinnliches oder mimetisches Begehren hervor, dem sich der*die Begehrende aber nicht bewusst zu stellen vermag, weil die kulturelle Disziplinierung dies untersagt. Dem psychoanalytischen Grundsatz folgend, nach dem Verdrängung bedeutet, dass die Repräsentation oder Symbolisierung des Triebwunsches aufgegeben wird, nicht aber der Wunsch und seine Energie, kommt es zu einer Verkehrung der Repräsentation, die nun über einen projektiven Vorgang dem zugesprochen wird, auf das eigentlich das eigene Begehren gerichtet ist. Über diese Veräußerung kann die Lust zugleich gelebt und bestraft werden, da man sie ja im Anderen verunglimpft und verfolgt.

So plausibel diese Erklärung für viele Situationen des Alltags und auch für die Strukturen rassistischer und sexistischer Ideologie zunächst ist, sie verliert an Plausibilität, wenn es um den Versuch geht, Exzesse der Gewalt und die Dynamiken der Verknüpfung von Sexualität und Gewalt zu erklären, wie sie in Situationen politischer Massengewalt regelmäßig auftreten. In dem ersten unserer beiden Beispiele schließt sich an den mit heftigen Beckenbewegung geäußerten Ruf „Fuck political correctness“ eine Folge von Äußerungen an, die die Verknüpfung symbolisch deutlich artikulieren. Im Zusammenschnitt der New York Times lautet die Folge: „Build that wall / Fuck those dirty beaners / Fuck political 
correctness / Fuck Islam / Hillary is a whore / Hang the bitch." Hier gibt es, wie gesagt, kein Objekt des Begehrens mehr, es geht um eine abstrakte Figur, in der eine Dynamik der Intimität vorherrscht: Über eine Abgrenzung, die die horizontale, nicht hierarchische Begegnung mit dem Anderen unterbinden möchte, über die rassistische Erniedrigung anderer Menschen mittels eines auf Reinlichkeit und Essensgewohnheiten bezogenen, also analen Schimpfwortes, dem die sexuell konnotierte Forderung der Penetration nachfolgt, über die schon diskutierte Abwertung und zugleich Überschreitung der Grenze eines ethischen Gebotes und die Abwertung und Überschreitung der Grenze zu einem ethnisch-religiösen Kollektiv bis hin zur direkten abwertenden Sexualisierung der damaligen Präsidentschaftskandidatin der Demokratischen Partei und dem abschließenden Aufruf zum Lynchmord. Die Objekte sind austauschbar, von ihnen geht keine eigentliche Aufforderung mehr aus. Wir haben es aber mit einer Kette von Äußerungen zu tun, die alle etwas mit Grenzziehungen und ihren Überschreitungen zu tun haben.

Wie kann man es psychologisch verstehen, wenn die Übertretung selbst als Ziel und Zweck von Äußerungen und Handlungen erscheint? Man kann diesen Vorgang nicht mehr vom Objekt her verstehen, sondern nur von der Szene her, der Narration oder der Infrastruktur, die er entwirft. Der Ausfall des Objektes, der Repräsentation eines Begehrens, eines Wunsches ist dabei prägend. Es findet eine direkte Koppelung oder Verlötung zwischen einer triebhaften Intensität und einer abstrakten Vorstellung statt, wobei in der abstrakten Vorstellung wiederum die Szene der Übertretung eingeschrieben ist: Sei es die Verhinderung eines Eindringens von etwas, das als Differenz vorgestellt wird, durch die Bildung einer Mauer; sei es, dass das Differente selbst mit analen Schimpfworten als etwas Grenzenloses imaginiert wird; sei es, dass der Anspruch der Anerkennung von Differenz und damit darauf, überhaupt als Objekt in Erscheinung treten zu können, direkt attackiert wird; sei es, dass in der Misogynie die geschlechtliche Differenz als sozialer Ausschluss dargestellt und mit der Aufforderung des Tötens verbunden wird. Oder am Beispiel der Zitate Trumps: die Aufforderung zur Körperverletzung, die Imagination des willkürlichen Mordes und die Inszenierung des Inzests.

Man kann diese Handlungen nicht von einer Annahme der Triebunterdrückung und des Ausagierens von verdrängten Triebfixierungen her verstehen. Es sind ja mit intensivem Genuss vorgetragene Szenen, es handelt sich um das Ausagieren eines Triebes, der nicht mit einem Imaginären verbunden ist, mit Bildern und Repräsentationen, sondern mit Phantasmen, mit Sprache ohne den Anspruch auf Repräsentation, ohne Bezug auf eine Welt, die eh differenzlos als mess bezeichnet wird. An die Stelle des Bezugs auf die Welt und ihre Objekte ist der Bezug auf Sprache und ideologische Formeln getreten. Die Äußerungen konturieren eine Infrastruktur, in der es keine Objekte mehr gibt, sondern eine Dramaturgie der Differenz, die allein durch die Figur der Übertretung selbst geschaffen wird. Es ist eine Infrastruktur, in der Triebintensität und Gesetz verschweißt sind. Das Gesetz hat dabei gleichsam zwei Seiten, durch deren Gleichgewicht es überhaupt gehalten wird: die Seite der Willkür und die Seite der Grenze, die gewissermaßen den letzten Rest eines Weltbezugs darstellt. Der Akt der Willkür, des Auslebens 
des Souveränitätsphantasmas, des übergroßen élan vital, ist notwendig verknüpft mit der Aggression, der Übertretung, der Gewalt gegen alles, was als Differenz auftreten und daran gemahnen könnte, dass die Größe des Eigenen an der Realität selbst eine Grenze haben könnte. Das Souveränitätsphantasma braucht die Vorstellung der Übertretung, um sich überhaupt als solches inszenieren zu können. Insoweit ist das, was man Gesetz nennen kann - im Unterschied selbstverständlich zum Recht, das einen diskursiven Raum der Auseinandersetzung eröffnet, in dem der Anspruch auf Anerkennung zumindest artikuliert werden kann -, immer an die Willkür gebunden.

\section{Fetisch: Ausstellen und Verbergen}

Die Übertretung muss ausgestellt, die soziale Tat selbst aber verleugnet werden. Anders gesagt: Der für die Inszenierung des Souveränitätsphantasmas notwendigen Übertretung darf keine Wahrnehmung der Wirklichkeit des Handelns entsprechen, sie darf nicht mit einer irgendwie befragbaren Repräsentation der Welt verbunden sein. Ist dieses Triebgeschehen noch mit dem Unterschied zwischen Verdrängung und Verleugnung zu fassen, wie ihn Freud verstand?

Zwischen der Verdrängung, die der Erklärung von Gewalt als projektiver Abwehr zugrunde liegt, und der Verleugnung besteht nach Freud der deutliche Unterschied, dass im Falle des Verdrängten eine unbewusste Objektfixierung bestehen bleibt, deren Bewusstwerdung eben im Vorgang der Projektion abgewehrt wird, im Falle der Verleugnung jedoch das Offensichtliche eher einer Spaltung unterliegt. In seiner späten Schrift „Abriß der Psychoanalyse“ von 1938 schreibt Freud, dass Verleugnungen der Wahrnehmung sehr häufig vorfallen,

„nicht nur beim Fetischisten, und wo immer wir in die Lage kommen, sie zu studieren, erweisen sie sich als halbe Maßregeln, unvollkommene Versuche der Ablösung von der Realität. Die Ablehnung wird jedesmal durch eine Anerkennung ergänzt, es stellen sich immer zwei gegensätzliche von einander unabhängige Einstellungen her, die den Tatbestand einer Ichspaltung ergeben“" (Freud 1938: 134-135).

Das Beispiel des Fetischisten, so Freud zuvor in seiner Argumentation, ist nur ein „,besonders günstiges Studienobjekt“ dieser Spaltung des Ich im Vorgang der Verleugnung. Der Fetisch produziert ein Objekt dort, wo es eigentlich einen Objektverlust, einen Mangel, eine Leere gibt. Das ist in einem weiteren Sinne genau das, was Freud in seinen Bemerkungen über den Fetischismus als den doppelten Glauben bezeichnet, dass das Weib einen Penis habe und auch nicht. Es wird in diesem Zusammenhang übrigens fast mit Erstaunen von Freud konstatiert, dass der Fetischist in der Regel keine Probleme habe, seinen Trieb auszuleben. Die Verleugnung der Kastration geschieht in diesem Sinne erfolgreich: der Fetisch ist „Zeichen des Triumphes über die Kastrationsdrohung und Schutz gegen sie“ (Freud 1927: 313). 
So sehr der Fetisch mit einem Bezug auf das Fehlen verknüpft ist, so dient er jedoch gerade dazu, die Erfahrung dieses Fehlens zu vermeiden. Er liegt also vor der Realisierung des Mangels, schützt durch eine Irrealisierung das Subjekt vor dieser Erfahrung. Ein Fetisch ist in diesem Sinne erfahrungslos und deshalb auch so schwer befragbar. Die seiner Infrastruktur folgenden Handlungen lassen sich nur schwer symbolisieren und mentalisieren. Ja, die Übertretung selbst ist der Fetisch, durch den sich die Willkür oder das Phantasma der Souveränität, das eben die eigene Bezüglichkeit zur Welt negiert, selbst erschafft: „All of this done out in the open as if by doing it publicly it was O.K. No one would object,“"kommentiert Michael Moore die Passage seines Films, in der es um die Ausstellung der sexualisierten Beziehung Trumps zu seiner Tochter und danach um das unbefugte Betreten der Kabine ging, in der Frauen sich gerade für einen Schönheitswettbewerb umziehen (Moore 2018: 0.24:34).

Dadurch, dass die Souveränitätsanmaßung an die Willkür der Übertretung gebunden ist, tendiert sie auch dazu, diese Übertretung immer neu zu inszenieren. Trumps Twitter-Account lässt sich dafür sehr effektiv gebrauchen, da in der auf Kürzel reduzierten Sprache gar keine diskursive Auseinandersetzung mehr möglich ist. Sie agiert aus, aber an beliebigen Gegenständen. Die Verleugnung der Welt - und das mag eine, wenn auch geringe Hoffnung begründen, dass wir in seiner Amtszeit nicht in einem nuklearen Krieg untergehen - muss keinen Charakter einnehmen, über den die Welt die merkwürdige Konsistenz eines Objektes der Negation erhält. Letzteres geschieht jedoch in den apokalyptischen Visionen eines Stephen K. Bannon und der Alt-Right. Erroll Morris hat in seinem Film American Dharma (2018) ein Porträt des Mannes gezeichnet, der über Jahre die Website Breitbart News Networks geleitet, maßgeblich zum Erfolg der Wahlkampagne Trumps beigetragen und nach Trumps Amtsantritt zunächst die Aufgabe eines Sonderberaters oder Chefstrategen übernommen hatte (Morris 2018). Morris lässt sich in diesem Porträt weit auf die affektive Infrastruktur der rechten Souveränitätsanmaßung ein. Sie ist mit der Szene der Zerstörung, des Krieges, der Auslöschung ebenso verbunden wie mit der des Helden und seiner Mission. Bannon selbst stellt diese Vorstellungen dabei an einer ganzen Reihe von Reinszenierungen von Szenen des US-amerikanischen Kinos dar, Szenen, die Bannon selbst ausgesucht hat und die zu seinen Lieblingsszenen gehören.

Verleugnung und Verdrängung führen zu unterschiedlichen Formen des Verhaltens und verlangen andere Weisen der Analyse. Denn während es bei der Verdrängung vor allem um Vorgänge der Verdichtung und Verschiebung geht sowie um projektive Abwehr, die auch als Zuschreibung der eigenen Handlung auf andere Akteure zu verstehen ist, wo sie dann bekämpft werden, sind Handlung oder Äußerung und psychodynamische Bedeutung im Falle der Verleugnung nicht mehr sprachlich aufeinander bezogen. Freud vergleicht die „Einsetzung des Fetisch“ mit dem Haltmachen der Erinnerung bei traumatischer Amnesie: „Auch hier bleibt das Interesse wie unterwegs stehen, wird etwa der letzte Eindruck vor dem unheimlichen, traumatischen, als Fetisch festgehalten" (Freud 1927: 315). Der Fetisch ist Markierung einer Spaltung, er derealisiert. Er ist meist auch nicht als Desymbolisierung zu verstehen, weil er schon vor der Symbolisierung oder 
Realisierung selbst schützt. Das begünstigt nicht nur den Triebgenuss, sondern auch die Aggressivität und zerstörerische Wirkung, denn die Wirkungen und Folgen der Handlungen werden wohl wahrgenommen, nicht aber realisiert, nicht als Handlungen angesehen, für die man verantwortlich sein könnte.

Freuds Beispiele für sexuelle Fetische - Füße, Schuhe, Pelz und Samt - versteht er gerade nicht als Symbolisierungen, sondern als eher zufällige Markierungen. Selbst der Pelz, der auf die Genitalbehaarung verweise, symbolisiert eben nicht das weibliche Geschlecht, sondern schützt im Gegenteil vor der Wahrnehmung desselben. Während verdrängte Dimensionen von Handlungen sich in gewissem Maße hermeneutisch lesen lassen, lassen sich also Handlungen, deren psychische Energie durch Verleugnung vor ihrer Wahrnehmung durch den Handelnden geschützt werden, nur durch Verfahren verstehen, die sich als szenische Vervollständigung beschreiben lassen. Das Verleugnete ist ja nicht etwas, das unsichtbar wäre. Es ist nur nicht adressierbar. Das Sichtbare und das Sagbare sind weit auseinandergetreten. Dieses Auseinandertreten steht im Zentrum dessen, was man das Obszöne nennt. Wenn man von der wahrscheinlichen Etymologie des Begriffs als etwas, das off-scene ist, her denkt, dann geht es nicht darum, dass das eine sichtbar ist und das andere nicht, sondern dass das Adressierbare und das Obszöne in zwei getrennten Räumen stattfinden: eben die Bühne oder Szene und die Narration. Im Obszönen des Alltags dürfte das Verhältnis von Sagbarkeit und Sichtbarkeit gerade anders als im Theater gewichtet sein: Obszön ist das, was auf der Bühne sichtbar, aber nicht sagbar ist. Es ist das, was ausgestellt wird, aber nicht adressiert werden kann. Trump adressiert seine Tochter so schamlos sexuell, dass es gar nicht als der Missbrauch wahrgenommen werden darf, der es doch so offensichtlich ist. Etwas Ähnliches lässt sich auch für die anderen angeführten Artikulationen Trumps sagen. Wenn er darauf wettet, dass er keine Wähler*innen verlieren würde, wenn er am helllichten Tage jemanden auf der 5th Avenue erschießt, spricht er ganz direkt dieses Verhältnis von Ausstellung und Verleugnung an, ja er inszeniert es. Und wenn er seine Zuhörer*innen dazu aufruft, etwaige Störer*innen zu verprügeln, fordert er offen zu einer Straftat auf, für die er doch zugleich das Einverständnis des Gesetzes verspricht.

Im Obszönen treten das Sichtbare und das Sagbare weit auseinander, der Diskurs aber, der das Sagbare bestimmt, ist in der Regel mächtiger als das Sichtbare, weil die Rede dem Subjekt eine Position zuweist und ermöglicht, die es im Sichtbaren nicht ohne weiteres hat. Die Positionierung des Subjekts im Sichtbaren ist abhängig von einer deiktischen Geste, die aber vor allem das Sprachliche der Rede oder des Körpers erzeugt. So kann die Sprache das, was sichtbar ist, regelrecht unsichtbar machen, wenn es durch den Diskurs nicht oder falsch kontextualisiert wird. Das führt zu einer bis ins Unheimliche reichenden Erfahrungslosigkeit, da ganze Vorgänge vollzogen werden, ohne dass das Subjekt sich angesprochen fühlt. Im Fetisch und im Obszönen kann etwas genossen werden, von dem man gar nicht wissen muss. Es findet im Bereich des Symbolischen oder Mentalen gar nicht statt.

Diese Überlegungen korrespondieren mit dem Verständnis des Obszönen, wie es in der psychoanalytischen Theorie von Jacques Lacan entworfen wird. Das 
Obszöne ist auch hier eng verbunden mit dem, was Lacan jouissance nennt und was sich mit relativ geringem Bedeutungsverlust als Genuss übersetzen lässt. Im Genuss überlässt sich das Ich nicht nur einer Lust, die ihm geschieht und die es nicht ohne weiteres kontrollieren kann. Es muss gar nicht wissen, dass es genießt. Man kann auch davon sprechen, dass sich das Ich im Genuss entgrenzt. Es verliert sich gewissermaßen in der Verknüpfung von Trieb und Gesetz. Lacan formuliert:

„So kommen wir zu der Formel, daß, um zu diesem Genuss zu gelangen, eine Übertretung notwendig ist und daß das Gesetz genau dazu dient. [...] Die Übertretung auf den Genuss hin geschieht allein, indem sie sich auf das entgegengesetzte Prinzip stützt, auf die Formen des Gesetzes“ (Lacan 1996: 215).

\section{Soziale Relation und Wahrheit - Dynamik der Fake News}

Wenn ein Fetisch an die Stelle eines verlorenen Dings tritt und damit sein Fehlen verleugnet wird, heißt das nicht schon, dass er als Objekt positiv bewertet gewesen sein muss. Gerade auch Szenen, die Gefühle der Furcht, Angst und Ohnmacht auslösen, können mit einem Fetisch verleugnet und gebunden werden. Was in dieser Spaltung ausfällt, ist eben das, was man als Erfahrung und Symbolisierung, als Bild, Diskursfähigkeit und intersubjektive Kommunikation bezeichnen könnte. Was ist passiert, wenn diese Dimension, die in der Lacanschen Begrifflichkeit vor allem mit dem Imaginären verbunden ist, gewissermaßen wegfällt und es eine direkte Koppelung von Realem und Symbolischen gibt? Ich will im Folgenden das nicht weiter in den Begriffen Lacans diskutieren, sondern entlang der psychoanalytischen Entwicklungspsychologie, wie sie von Peter Fonagy und seiner Forschungsgruppe entfaltet wurde. Mit ihr scheint es mir möglich, die Schwierigkeit der Entwicklung des Imaginären oder, in Fonagys Begriffen, der Mentalisierung komplex zu beschreiben. Sie bietet damit auch eine Möglichkeit, zu erfassen, wie das Verhältnis von Symbolisierung und Weltbezug dynamisch zu verstehen ist. $\mathrm{Zu}$ beachten ist dabei, dass der Begriff der Symbolisierung gerade nicht primär in dem Bereich anzusiedeln wäre, den Lacan die symbolische Ordnung nennt, sondern eben im Imaginären, im Reich der Einbildungskraft und der ästhetischen Erfahrung. Meine an Fonagy angelehnte These dabei ist, dass es zur Herausbildung einer Fähigkeit zur Symbolisierung einer Erfahrung einer sowohl dynamischen wie gesicherten Beziehung zur Welt bedarf und dass Kampagnen wie etwa die von Trump verfolgte Strategie der Fake News gerade darauf zielen, diesen Raum der Symbolisierung zu zerstören.

Gegenüber den klassischen psychoanalytischen Ansätzen Freuds setzen die Überlegungen Fonagys stärker auf den primär sozialen Charakter der Interaktion. Aus ihr bilden sich überhaupt erst Differenzen wie die zwischen dem eigenen Denken und dem der Anderen und zwischen dem eigenen Denken und der Welt heraus. Eine gewisse Wendung zu den Strukturen der Interaktion hatte schon die in den Jahren nach dem Zweiten Weltkrieg entwickelte Bindungstheorie von John Bowlby vollzogen. Dabei fragt Bowlby allerdings zunächst nicht nach der 
Entstehung der Pole des Selbst und des Anderen und geht zumindest anfangs von einer direkten Beziehung zwischen der Interaktion des Kindes mit seinen Bezugspersonen und der Entwicklung des Verhaltens zu anderen Personen und ihm fremden Umwelten aus. Demnach bestimmen die Reaktionen der zentralen Bezugspersonen auf das Verhalten des Kindes in einem hohen Maße die Beziehungen des Kindes zu anderen Personen und zur Welt. Sind diese vertrauensvoll und sicher, kann sich das Kind auch auf ihm Fremdes zubewegen. Auch wenn diese Narration darin etwas zu schematisch ist, dass sie die Komplexität der Reaktionen des Kindes und seines inneren Erlebens unterschätzt, lassen sich einige Annahmen der Bindungstheorie in das komplexere psychoanalytische Verständnis der Psyche integrieren. Die zentrale Brücke besteht in der Beobachtung, dass sich das Selbst des Kindes durch die Interaktion mit den Bezugspersonen entwickelt, und zwar in der Weise, dass das Kind die mentalen Annahmen der Bezugspersonen über sich letztlich auf sich selbst bezieht und in seiner spezifischen Perspektivierung verändert annimmt. Diese Entstehung eines Ichs oder eines Bewusstseins der eigenen Subjektivität über die Interaktion mit den Bezugspersonen bringt es mit sich, dass das Kind zunächst davon ausgeht, dass sich die eigene Wahrnehmung der Welt und die der Menschen, mit denen es zusammen ist, nicht unterscheiden. Was das Kind wahrnimmt, wird auch von den Anderen so gesehen. Folglich kann es aber auch kein Bewusstsein dafür geben, dass der eigene Blick auf die Welt subjektiv ist, dass die Welt anders ist als das, was man selbst glaubt, und dass andere Menschen etwas anderes denken und sehen können als man selbst. Peter Fonagy und Mary Target nennen diese Weise der Erfahrung den Äquivalenzmodus (Fonagy/Target 2007). Gleichzeitig entwickelt das Kind aber zunehmend auch die Fähigkeit des Spiels. Im Spiel treten die Dinge und ihre Bedeutung auseinander, die Dinge werden Medium. Freud hatte diesen Vorgang eine ,große kulturelle Leistung“ genannt, als er beschrieb, wie sein Enkelkind mit einer Spule spielte und in dieser Weise die Erfahrung von An- und Abwesenheit seiner Mutter symbolisierte (Freud 1920: 13). Dieses Spiel kann aber leicht etwas Haltloses und Bedrohliches bekommen, da das Kind ja in eine eigene Welt taucht, die ihre Bestimmtheit schnell verlieren und die auch ebenso schnell in ein Gefühl der Ohnmacht umschlagen kann.

Es ist, so Fonagy/Target, eine bekannte Beobachtung, dass Kinder oft sehr viel mehr Zeit damit verbringen, die Regeln des Spiels auszuhandeln als mit dem Spiel selbst. Es gilt, die Gefahren des Selbstverlusts im Spiel zu begrenzen und ein Einvernehmen über den intersubjektiven Charakter des Tuns auszuhandeln. Gelingt dieser pretend mode, dieser Als-ob-Modus, kann es zu einem intensiven sozialen Erleben kommen. Für Fonagy und Target ist es nun das gelingende Zusammenspiel dieser Erfahrungsmodi der Äquivalenz und des Als-ob, die es ermöglichen, die Erfahrung der Subjektivität jeder Welterfahrung zu machen oder auch zuzulassen. Denn zunächst handelt es sich ja um eine immense Einschränkung der eigenen Welt und der Macht über sie, wenn das Kind realisieren muss, dass die eigene Sicht auf die Welt beschränkt und verzerrt ist. Allerdings ist diese Einsicht die Bedingung dafür, den Blick und die Meinungen anderer Menschen als 
ihre persönlichen mentalen Vorgänge ebenso ansehen zu können wie die eigenen Gedanken. Dieser Prozess wird von Fonagy/Target Mentalisierung genannt.

Die selbstbezogenen, sich im Modus der Souveränitätsanmaßung bewegenden Handlungsweisen Trumps sind demzufolge als Ausfall der Mentalisierung zu beschreiben. Die Differenzierung zwischen dem eigenen Denken und der Welt anzuerkennen, fällt dem gegenwärtigen US-amerikanischen Präsidenten offensichtlich schwer. Wenn etwas nicht stimmt, dann ist es nicht die eigene Sicht auf die Welt, sondern es ist die Welt, die schlecht ist: Dazu gehört auch die Unfähigkeit, sich mit Kritik auseinandersetzen zu können, die ja bis zur jede demokratische Legitimation überschreitenden Anmaßung geht, für selbst begangene Vergehen eine Amnestie aussprechen zu können. Der Äquivalenzmodus dominiert die Twitter-Äußerungen Trumps. Das Medium sichert aufgrund seiner kommunikativen Struktur und seiner Verbreitung gleichsam zu, die eigenen Äußerungen als immer schon von anderen geteilt anzusehen.

Worauf es mir aber in diesem Zusammenhang besonders ankommt und was mir auch für die kulturellen Veränderungen, die dadurch bewirkt werden, noch nachhaltiger erscheint, ist die Fake-News-Kampagne, mit der neu-rechte Politik mittlerweile weltweit arbeitet. Sie erscheint wie ein kindlicher Als-ob-Modus und erlaubt auch eine hohe Intensität an Genuss. Verschiedentlich ist behauptet worden, es gäbe eine Verbindung zwischen der Kritik an Wahrheitsansprüchen, wie sie im Kontext des Poststrukturalismus, der Kritik der Episteme und des Diskurses durch Michel Foucault und die Dekonstruktion entwickelt wurde, und der Politik der Rechten und ihrer Behauptung, es würden in den Medien ,Lügen“ verbreitet. Keine der erwähnten Kritiken zielte jedoch auf eine Gleichstellung von Wahrheitsansprüchen mit Lügen. Worum es etwa bei Foucault ging, war ja gerade der Nachweis, dass Wahrheitsansprüche und ihre sie tragenden Episteme und Diskurse sozialer und politischer Natur sind. Sie dienen der Verständigung über Weltzusammenhänge und regeln die Konkurrenz über Interessen. Der Widerstand gegen Wahrheitsansprüche geschieht nicht von einem Außen aus, er geschieht in den Diskursen selbst. Sie bieten so etwas wie einen gemeinsamen Grund, was nicht schon bedeutet, dass ein herrschaftsfreier Dialog möglich wäre. Aber gibt eine Gesellschaft den Anspruch einer Verständigung über die Wirklichkeit auf, verliert sie auch den gemeinsamen Raum der Kommunikation und läuft auf eine Situation der Willkür und der offenen Gewalt zu.

Es geht bei der Wahrheit nicht um etwas, das hinter einem Vorhang verborgen ist, sondern um ein soziales Gut, einen kulturellen Reichtum. In der Möglichkeit der Auseinandersetzung mit Wahrheitsansprüchen steckt immer ein Stück Anerkennung der Differenz zwischen dem eigenen Denken und der Welt. Es geht im Kern um Mentalisierung, um die Einsicht und Anerkennung, dass das eigene Denken ebenso wie das der Anderen ein mentales Geschehen ist. Das erlaubt nicht nur, über diskursive Verfahren zu Kompromissen zwischen gegenläufigen Interessen zu kommen. Eine Gesellschaft, in der es ein Miteinander verschiedener sozialer und kultureller Gruppen gibt, setzt einen mentalen Raum voraus, in dem das eigene Denken und Fühlen ebenso wie das des Anderen als ein subjektives Erleben verstanden werden kann. Mentale Räume in diesem Sinne sind grundsätzlich auch 
ästhetischer Natur. Sie können als ästhetische ebenso wie als diskursive Symbolisierungen verstanden werden. Ästhetische Erfahrung ist von einer Präsenz von Differenzen an ein und demselben Ort bestimmt. Ohne einen solchen kulturellen Raum bilden sich Gemeinschaften vornehmlich über das Prinzip der Homogenisierung, das mit dem Ausschluss von Differenz einhergeht. Das führt zu sozialen Spaltungen und Entsolidarisierungen und unterläuft damit auch das soziale Potenzial des Widerstandes gegen Enteignung und Ausbeutung. Die neoliberale Wirtschaftspolitik benötigt von daher die Strategien der Entsolidarisierung bzw. der Schwächung und partiellen Zerstörung des mentalen Raumes. Das ist die kulturelle Seite dessen, was Naomi Klein eher aus ökonomischer Sicht als disaster capitalism beschrieben hat (Klein 2007). Beides geht Hand in Hand. Die ökonomischen und ökologischen Katastrophen, die der Kapitalismus zur Aneignung von materiellen Ressourcen nutzt, unterlaufen ein Vertrauen in die Welt, das mit dem Vertrauen in die Welt, welches sich über Wahrheitsdiskurse einstellt, korrespondiert.

Die Fake-News-Kampagne ist ein effektives Werkzeug der Diffundierung und Enteignung des Gemeingutes Wahrheit. Der sozialpsychologische Hintergrund ihrer Wirksamkeit ist nun wiederum in der Fragilität des mentalen Raumes zu suchen. Fonagy und Elizabeth Allison gehen davon aus, dass Wahrheit eng mit dem Vertrauen verbunden ist, das Kinder in die Welt entwickeln können (Allison 2016). Letzteres hängt sowohl davon ab, ob sich ein Kind des Bezugs zu seinen Betreuungspersonen in dem Sinne sicher ist, als es ihn als eine mentale Verbindung erfahren kann, ein joining of minds, als auch davon, ob es zwischen den mentalen Prozessen und der Welt unterscheiden kann. Ein Vertrauen in das, was man als Wissen von den Bezugspersonen überliefert bekommt - Fonagy/Allison nennen es epistemic trust - ist eine Notwendigkeit des Überlebens (Fonagy/Luyten/Allison 2015: 584). Aber genau dieses epistemische Vertrauen ist, wie verschiedene Experimente gezeigt haben, eng verbunden mit dem Vertrauen in die Personen, die das Wissen übermitteln. Dabei handelt es sich nicht um eine einfache Aufnahme oder Imitation, das Vertrauen hat sehr viel damit zu tun, dass die Bezugspersonen auch für das einstehen, was sie sagen. Mentalisierung ist ,the capacity of one individual to understand the actions of another in terms of the thoughts, feelings, wishes, and desires“ (ebd.: 586). Die Verknüpfung von Äußerungen mit dem Selbst werden mittels ,ostentive cues“, also etwa herausstellender Zeichen oder Zuschreibungen, in der Kommunikation vollzogen (ebd.).

Der Mangel an epistemischem Vertrauen führt nun schnell dazu, dass die Welt selbst als etwas gesehen wird, das von Verschwörung beherrscht wird. Sie wird gleichsam zu einem Produkt von Menschen, denen man nicht vertrauen kann. Nicht nur für das, mit dem man sich nicht konfrontieren und dessen Folgen man nicht sehen will, wird eine Ursache in geheimen Verabredungen und Verbindungen gesehen, das Vertrauen in die Welt insgesamt ist so fragil, dass die Nachrichten über die Welt selbst als eine Verschwörung gesehen werden. Die Kategorisierung aller kritischen und unabhängigen Berichterstattung als ,Lügenpresse" drückt eben dies aus. Sie mag einerseits entlasten, andererseits ist sie aber ein sich selbst performativ antreibender Vorgang, weil er die Spiralen der 
Verschwörungstheorie antreibt. Jeder Verschwörungstheorie unterliegt die Lust, selbst die Welt in der Verschwörung zu verkaufen. Die neoliberalen Eliten können sich diese aus den Konturen geratene Welt so tatsächlich immer weiter aneignen, den meisten Anhänger*innen des rechten Populismus bleibt der Genuss an der souveränen Geste der Zerstörung des Vertrauens in die Welt. Die Möglichkeit, dass in der Verbindung des Äquivalenzmodus und des Als-ob-Modus eine Ko-Emergenz von Mentalisierung und Anerkennung der Eigenständigkeit der Welt steckt, kommt nicht zur Realisierung. Dass die eigene Sicht auf die Welt etwas Mentales ist, wird im Bezug auf die Ideologie der Gruppe zurückgenommen, das Als-ob, das sich im Zurückdrängen des Bewusstseins ausdrückt, dass es sich vielleicht doch so verhalten könne, dass es zum Beispiel einen Klimawandel gibt, kann dann in der Anmaßung der Souveränität überspielt werden. Die Spielregeln, über die sich die Kinder so lange unterhalten, sind zu Stammtischgesprächen und Twitter-Erklärungen geworden.

\section{Literatur}

Allison, Elisabeth/Fonagy, Peter: When is truth relevant? In: Psychoanalytic Quarterly 85/2 (2016): 275-303.

Berlant, Lauren: Cruel Optimism. Durham/London 2011.

Berlant, Lauren: The commons: Infrastructures for troubling times. In: Environment and Planning D: Society and Space 34/3 (2016): 393-419.

Berlant, Lauren: Big Man. In: Social Text Online (19.01.2017), https://socialtextjournal.org/big$\mathrm{man} /(15.01 .2019)$.

Bernstein, Richard: The rising hegemony of the politically correct. In: The New York Times (28.10.1990), https://www.nytimes.com/1990/10/28/weekinreview/ideas-trends-the-rising-hegemony-of-the-politically-correct.html?mtrref=www.google.com\&gwh=F79FDEE67B6991AFDEEFE535ADBBD661\&gwt=pay (14.01.2019).

Bush, George H.W.: University of Michigan Commencement Speech (04.05.1991), https://www. youtube.com/watch?v=4IORaF6fi_Y (14.01.2019).

Diamond, Jeremy: Trump: I could , shoot somebody and I wouldn't lose voters'. In: CNN politics (24.01.2016), https://edition.cnn.com/2016/01/23/politics/donald-trump-shoot-somebodysupport/index.html (15.01.2019).

Fanon, Frantz: Die Verdammten dieser Erde. Frankfurt a. M. 1981.

Fonagy, Peter/Target, Mary: Playing with reality IV. A theory of external reality rooted in intersubjectivity. In: International Journal of Psychoanalysis 88/4 (2007): 917-37.

Fonagy, Peter/Luyten, Patrick/Allison, Elizabeth: Epistemic petrification and the restauration of epistemic trust: A new conceptualization of borderline personality disorder and its psychosocial treatment. In: Journal of Personality Disorders 29/5 (2015): 575-609.

Freud, Sigmund: Abriss der Psychoanalyse [1938]. In: Ders.: Gesammelte Werke XVII: Schriften aus dem Nachlass. Frankfurt a. M. 1955: 64-140.

Freud, Sigmund: Jenseits des Lustprinzips [1920]. In: Ders.: Gesammelte Werke XIII: Werke aus den Jahren 1920-1924. Frankfurt a. M. 1967: 3-72.

Freud, Sigmund: Fetischismus [1927]. In: Ders.: Gesammelte Werke XIV: Werke aus den Jahren 1925-1931. Frankfurt a. M. 1967: 311-320.

Horkheimer, Max/Adorno, Theodor W.: Dialektik der Aufklärung. Philosophische Fragmente. Frankfurt a. M. 1983.

Klein, Naomi: The Shock Doctrine: The Rise of Disaster Capitalism. Toronto 2007.

Lacan, Jacques: Die Ethik der Psychoanalyse. Das Seminar VII. Weinheim/Berlin 1996. 
Mbembe, Achille: Necropolitics. In: Public Culture 15/1 (2003): 11-40.

Mbembe, Achille: Politiques de l'inimitié. Paris 2016.

Parker, Ashley/Corasaniti, Nick/Berenstein, Erica: Voices from Donald Trump's rallies, uncensored. In: The New York Times (03.08.2016), https://www.nytimes.com/2016/08/04/us/politics/ donald-trump-supporters.html (14.01.2019).

Ross, Andrew/Diawara, Manthia/Doty, Alexander/Lubiano, Wahneema/Rose, Tricia/Shohat, Ella/ Spigel, Lynn/Stam, Robert/Wallace, Michele: A symposium on popular culture and political correctness. In: Social Text, 36 (1993): 1-39.

Schmidt, Caroline/Schiele, Katharina: Demo in Dresden: Pegida vs. TV-Team. In: Zapp Das Medienmagazin (22.08.2018), https://www.youtube.com/watch?v=7n9iy5DwTm8 (14.01.2019).

Trump, Donald: Interview with David Muir. In: ABC News (26.01.2017), https://www.youtube. $\mathrm{com} / \mathrm{watch}$ ? $\mathrm{v}=$ SKEz7_TITZA (15.01.2019).

Weigel, Moira: Political correctness: how the right invented a phantom enemy. In: The Guardian (30.11.2016), https://www.theguardian.com/us-news/2016/nov/30/political-correctnesshow-the-right-invented-phantom-enemy-donald-trump.

\section{Filme}

American Dharma (2018). USA 2018, Regie: Morris, Errol, Fourth Floor Productions.

Fahrenheit 11/9 (2018). USA 2018, Regie: Michael Moore, Midwestern Films.

Open Access Dieses Kapitel wird unter der Creative Commons Namensnennung 4.0 International Lizenz (http://creativecommons.org/licenses/by/4.0/deed.de) veröffentlicht, welche die Nutzung, Vervielfältigung, Bearbeitung, Verbreitung und Wiedergabe in jeglichem Medium und Format erlaubt, sofern Sie den/die ursprünglichen Autor(en) und die Quelle ordnungsgemäß nennen, einen Link zur Creative Commons Lizenz beifügen und angeben, ob Änderungen vorgenommen wurden.

Die in diesem Kapitel enthaltenen Bilder und sonstiges Drittmaterial unterliegen ebenfalls der genannten Creative Commons Lizenz, sofern sich aus der Abbildungslegende nichts anderes ergibt. Sofern das betreffende Material nicht unter der genannten Creative Commons Lizenz steht und die betreffende Handlung nicht nach gesetzlichen Vorschriften erlaubt ist, ist für die oben aufgeführten Weiterverwendungen des Materials die Einwilligung des jeweiligen Rechteinhabers einzuholen. 